\title{
Productos andinos para el desarrollo de una gastronomía nacional
}

\author{
Andinos profucts for a national \\ gastronomy development
}

\begin{abstract}
JUAN MANUEL MONTES PÉREZ
(1D) https://orcid.org/0000-0002-1399-5719

Fundación Universitaria San Mateo (Colombia) / jmmontes@sanmateo.edu.co

LUISA FERNANDA DAZA CASTIBLANCO

Fundación Universitaria San Mateo (Colombia) / Ifernandadaza@sanmateo.edu.co

LIZZETH MARGARITA ANGARITA BÁEZ Fundación Universitaria San Mateo (Colombia) / Imangarita@sanmateo.edu.co
\end{abstract}

Artículo de investigación

Recepción: 09 de diciembre de 2020

Aceptación: 16 de marzo de 2021

Cómo citar este artículo

Montes Pérez, J., Daza Castiblanco, L., y Angarita Báez, L. (2020).

Productos andinos para el desarrollo de una gastronomía nacional. Sosquua 2(2), pp. 59-69. Recuperado a partir de: http:// cipres.sanmateo.edu.co/index.php/sosquua 


\section{Resumen:}

Hablar sobre los cultivos de la Región Andina puede llegar a considerarse como un tema extenso debido a la información limitada que se tiene sobre estos. Uno de los múltiples ejemplos son los cultivos de tubérculos o papas nativas en Colombia los cuales genera un conocimiento básico de los productos más tradicionales como la papa criolla, la sabanera y la pastusa. Por ello, esta investigación realiza una indagación y contextualización para dar a conocer al lector acerca de otros tubérculos o papas nativas como las ibias, los ollucos y la papa ratona morada, junto con sus diferentes usos y preparaciones en la gastronomía. Además, busca concientizar sobre la existencia de otras variedades de cultivos; no solamente las que comúnmente se consiguen en las tiendas o supermercados; también existen de diversos colores, formas y tamaños.

Palabras clave: tubérculos; región Andina; papas nativas; cultivo.

\section{Abstract:}

Talking about the Andean Region crops can be considered as an extensive topic due to the limited information that is available about these. One of the many examples are the crops of tubers or native potatoes in Colombia. It generates a basic knowledge of the most traditional products such as potato creole, savannah and pastusa. Then, this research inquires and contextualizes about other tubers or native potatoes such as: ibias, ollucos and papa ratona morada; as well as their different uses and preparations in gastronomy. As a result, it seeks to raise awareness that there are more varieties of crops; not only those that are commonly found in stores or supermarkets that exist in different colors, shapes and sizes.

Keywords: tubers; Andean region; native potatoes; crop. 


\section{Introducción}

Luchar por lo autóctono es luchar por lo de nosotros, no hay necesidad de traer semillas de otros lugares, ya que aquí se cuenta con semillas que son ancestrales, naturales y sin ningún riesgo para la salud.

Briceño, 2017

La gastronomía avanza, se transforma y se renueva; busca actualmente modernizarse, ir a la vanguardia, trabajar de la mano con la creatividad y la innovación. El propósito es buscar aumentar el conocimiento de las técnicas, métodos y resultados en las cocciones; así como generar nuevas experiencias para el comensal.

Sin embargo, en un ámbito cultural como en el que se encuentra Colombia, esta gastronomía autóctona procura a desaparecer (Rodríguez Arias, 2020). En ese sentido, desdeña las tradiciones y la cocina ancestral; además, deja de lado los métodos que habían pasado de generación en generación. Asimismo, desprecia las técnicas, los productos y recetas de la abuela para adoptar tendencias extranjeras.

Es el caso de los tubérculos, como los diversos tipos de papa y las raíces, que levantaron con estomago fuerte a los jóvenes que luego salieron a luchar por tierras abundantes llenas de fertilidad. Por tanto, con el paso de los años se fueron olvidando y los cultivos se convirtieron en el escaso conocimiento coloquial de tres (3) a cinco (5) clases de papa (pastusa, criolla, sabanera, superior y R12). En consecuencia, deja atrás un sinfín de productos como la ruba (olluco), papa ratona, ibia (oca), entre otros. De esta manera, están a punto de extinguirse por falta de divulgación e interés para trasmitir este conocimiento.

Al retomar las tradiciones de cocina de nuestros antepasados, se recupera una parte de la identidad propia, la cual se ha ido desvaneciendo en el tiempo. Entonces, este documento prioriza la conservación de la cocina tradicional, las costumbres y la identidad (Barón, Clavijo y Combariza 2010).

La cocina tradicional y ancestral ha perdido protagonismo en los últimos años; se ha convertido en un segundo plano, junto a los insumos y materias primas proporcionados por la tierra colombiana. Por esta razón, el presente trabajo tiene como objetivo exponer por medio de la investigación la importancia de algunos tubérculos poco usados. Asimismo, indicar determinadas recetas tradicionales utilizadas con estos productos y posteriormente llevarlo a la práctica en una composición final, empleando otros insumos.

El trabajo investigativo se tomó desde la perspectiva de aplicación tecnológica. Buscó no solo generar conocimiento; además, promover la praxis dentro del mismo con el fin de impulsar un impacto positivo en el sector agroecológico, evitando la pérdida de estos cultivos poco notables. Para la ejecución del documento se trabajó el método histórico, examinando archivos sobre la cultura de la región Andina. Se realizó la búsqueda de referencias tradicionales sobre el uso de los tubérculos en cuestión. También 
se utilizó el método analítico de forma que se detalló el origen de cada tubérculo, así como las características físico-químicas y organolépticas de cada uno.

Por ello, es necesario definir el tubérculo como la parte de un tallo subterráneo o de una raíz que se desarrolla y se engruesa por acumular en sus células sustancias de reserva. Busca representar y dar a conocer las diferentes formas de alimentación de los campesinos. A través de esto, la investigación llega a la creación de un proyecto que comparta de una manera más activa el uso y las características que brindan estos productos. Además, evidencia las diferentes preparaciones que se logran rescatar y adaptar con cada uno de los alimentos que contiene este documento.

Las recetas ancestrales como eje cultural de la historia marcan una pauta importante a la hora de crear. Por medio de estas se puede direccionar el uso de las materias primas. Esto se convierte en un punto de interés para la creación de nuevos productos e investigaciones culinarias. El objetivo es lograr el aumento en el consumo de los tubérculos por medio de diferentes técnicas, mezclas y sabores, que sean usados ampliamente en la gastronomía colombiana.

Al investigar sobre las características organolépticas de las ibias, olluco y papa ratona se pudo identificar que este puede ser un alimento de baja diversificación. Lo anterior, pues solo se han optado por realizar una mínima cantidad de platillos a lo largo y ancho de la región cundiboyacense. Esto se ve reflejado en que el impacto y uso dentro de la gastronomía es muy precario. Por ello, se ve la necesidad de ampliar y abrir puertas al desarrollo y reconocimiento a nivel culinario de ingredientes que sean utilizados de muchas más formas; así ampliando el campo de acción de los tubérculos.

\section{Ibia}

Oxalis tuberosa Molina conocida como oca en Ecuador, Bolivia y Perú, así como Ibia en Colombia; se caracteriza por ser un tubérculo apreciado por sus diversidades en colores y sabores particulares. Es una herbácea, de sabores dulces y consistencia harinosa, según los ejemplares depositados en el Herbario Nacional Colombiano. Sus principales cultivos se encuentran distribuidos por Nariño, Cauca, Cundinamarca y Boyacá. Son cultivadas a una altitud entre los 2800 y 4000 metros sobre el nivel del mar (msnm) y es considerada un patrimonio cultural heredado por Paeces, Muiscas y Laches (Rosero, 2010; Clavijo y Pérez, 2014).

Son raíces comestibles, muy tiernas y harinosas. Los indígenas las comen verdes, asadas y cocidas. También partidas por medio a la larga y las secan al sol para guardar. Son de buen sabor, algo dulces, como higos pasados (Cobo, 1890, citado Colombia Explicada, 2015).

Entonces, su consumo se hizo imprescindible debido a sus múltiples beneficios pues las hojas y ramas eran cocidas y comidas en tiempo de peste 
o de tabardillos. Lo anterior, para preservarse de todo contagio; su zumo se aprovecha contra toda inflamación y contra el incendio o intemperie de la gota; también en contra de la sordera, dispensando algunas gotas al oído. Asimismo, el cocimiento de las hojas con azúcar (o hecho jarabe), tomado en ayunas, templa la cólera y sangre. Contra el ardor de la orina ayuda a controlar las fiebres; además ayuda contra la inflamación de la garganta, dolores abdominales y problemas intestinales.

\section{Papa ratona morada}

En Ventaquemada, Boyacá, a manos de Pedro Briceño se han logrado rescatar alrededor de 40 tipos de papa en diez (10) años. Estas son compradas por 15 restaurantes aproximadamente. La gran mayoría de los nombres fueron rescatados junto con la especie y son referentes de animales y plantas (propio de la cultura muisca) Redacción nacional (2017).

Su especie es Solanum tuberosum Grupo Andigenum 2X y su nombre común es "Papa ratona" o "Ratona morada". Existen nueve (9) especies de papa ratona identificadas alrededor de Nariño. Entre estas, tres (3) tipos de ratona morada, tres (3) tipos de ratona roja, dos (2) tipos de ratona rosada y un (1) tipo denominado "Ratona". Estas clasificaciones se realizan con el fin de identificar la cantidad de almidones, descripción morfológica y organoléptica.

La papa ratona morada se caracteriza por su flor morada y blanca. Puede tener una floración profusa o escasa. El color externo es morado y el interno puede ser ausente o con vetas moradas. Finalmente, las cocciones idóneas para este producto son sopas, gracias a la cantidad de almidón que resulta en una textura harinosa y tierna.

\section{Olluco}

No se encuentra definido aún el lugar exacto del origen del olluco. Herrera, citado en León (1994), indica que se han observado plantas consideradas como silvestres en Perú (departamento del Cusco) y Bolivia. Así, se han encontrado de 50 a 70 clones. Por otro lado, King (1986) indica que el Olluco está entre las plantas domesticadas en los Andes, alrededor de 5500 A.C. Por lo tanto, se puede inferir que la zona Andina es el origen del olluco.

En 1948 el olluco migró a Europa, después de la escasez de papa, como cultivo alternativo potencial (King, 1988). Sin embargo, de acuerdo con Rousi et al. (1989), citado en Clavijo y Pérez (2014), se perdió el interés en el cultivo por sus bajos rendimientos. Hasta la fecha, ninguna variedad de olluco ha sido introducida en América del Norte, para la producción de tubérculos en condiciones naturales de campo. 
Actualmente, se cultiva en todos los países andinos. Desde Argentina hasta Venezuela, así como en muchos países como Finlandia, Francia y Nueva Zelandia (Avalos, 2008, p.42-47) Refiere SIEA (2012). Dentro de sus características nutricionales se constituye como fuente de calorías y proteínas para cerca de nueve (9) millones de personas que viven entre 2500 a $4000 \mathrm{msnm}$. El olluco presenta una variedad de usos culinarios, siendo consumido tanto en fresco, hervido, como deshidratado, en sopas y harinas (León, 1964). Las hojas suelen consumirse en ensaladas en Colombia y Perú debido a que contienen un 12\% de proteína y seis (6) de los ocho (8) aminoácidos esenciales en la dieta humana (Lescano, 1994; Barrera et al., 2004).

\section{Metodología}

La metodología empleada para la investigación tomó como referencia un rumbo analítico e histórico. Por medio del primer método se examinó y planteó las diversas características organolépticas que pudo llegar a tomar el producto; así, exponiéndolo bajo diferentes preparaciones, técnicas y modos de cocción. El segundo se evidencia dentro del trabajo a partir de diversos archivos estudiados y citados, posterior a la extracción de información relevante como origen, tradición y ancestralidad de cada producto.

Además, se manejó el método cualitativo, pues la información recolectada no obtuvo alguna variable de posición o análisis dentro del mercado. La población que se utilizó para el proyecto se dividió en dos (2). La primera fueron los campesinos debido a que ellos son quienes sienten directamente el impacto frente al olvido o erradicación de estos cultivos tradicionales. El segundo fue visto desde la perspectiva del estudiante; aquel que puede realizar un cambio si tiene el conocimiento pertinente sobre estos productos. Por esta razón, dentro del proyecto también se buscan alternativas innovadoras sobre el uso de estos tubérculos.

Finalmente, los instrumentos de investigación se basaron en tres (3) libros principales: Guía del campo de los cultivos andinos, Catalogo de papas nativas de Nariño, Colombia y el Recetario de tubérculos andinos de Turmequé y Ventaquemada Boyacá, Colombia. Fueron publicados por la FAO, la Universidad Nacional de Colombia y la Pontificia Universidad Javeriana respectivamente.

La investigación contó con la orientación de Pedro Briceño, campesino de la Región Andina (más específicamente de Nariño). Desde el 2008, Briceño lidera la recuperación del cultivo de papa nativa. Por tanto, por medio de comunicación telefónica realizó seguimiento de la investigación y brindó importantes aportes a la misma.

También contó con el apoyo de las estudiantes Lizzeth Margarita Angarita Báez, quien al comienzo del proyecto investigativo cursaba su último semestre como profesional en gastronomía. Asimismo, participó la estudiante Luisa Fernanda Daza Castiblanco quien actualmente se encuentra cursando séptimo (7) semestre de gastronomía. Este equipo de trabajo, bajo la supervisión del docente Juan Manuel Montes Pérez, se encargó de indagar, redactar y producir el resultado de la investigación. 


\section{Material de indagación.}

\section{Guía de campo de los cultivos andinos.}

El texto es una guía orientada a la extensión agrícola, con información útil a los agricultores y asistentes técnicos en la región andina; relaciona 20 cultivos andinos nativos y sus variedades. La guía contiene el resultado de numerosas investigaciones en una forma práctica y aplicable. Asimismo, revalora la cultura local y sus aportes de técnicas tradicionales, como el uso de variedades, la densidad de siembra, el manejo del suelo y la fertilización.

\section{Catálogo de papas nativas de Nariño, Colombia.}

Nace a partir del trabajo de indígenas y pequeños agricultores de la zona papera de Nariño, en el marco del proyecto "Mejoramiento de la producción de papa para contribuir a la seguridad alimentaria y nutricional de comunidades nativas en Colombia". Dichos agentes han cuidado y preservado por generaciones distintas semillas de papas nativas que se han ido adaptando a los cambios climáticos y de suelos. Este catálogo muestra todas estas semillas por medio de registro fotográfico y se especializó en documentar la información organoléptica, morfológica, cosecha, poscosecha y usos culinarios de los mismos.

\section{Recetario/tubérculos andinos de Turmequé y Ventaquemada, Boyacá, Colombia.}

Es parte clave en este proceso de conservación y un producto importante del proyecto "Diseño participativo de alternativas sostenibles de manejo y conservación de la agro biodiversidad de tubérculos andinos en el Departamento de Boyacá, Colombia" ejecutado por la Pontificia Universidad Javeriana (PUJ), la Corporación para el Desarrollo Participativo y Sostenible de los Pequeños Productores Rurales (Corporación PBA) y un grupo voluntario de 30 agricultores de los municipios de Turmequé y Ventaquemada. Es realizado en el marco del Consorcio Andino de innovación participativa.

Su elaboración contó con la participación activa de un grupo de familias de los dos (2) municipios, motivados por mantener el cultivo y el consumo del cubio, la ibia y la ruba. El conocimiento local de las diferentes formas de consumo fue muy importante en este proceso; constó de un diálogo de saberes y posibilitó la recuperación de diversas preparaciones familiares. Además, el aprender haciendo permitió la experimentación de las preparaciones y la validación de otras, encontradas en recetarios de diferentes regiones y países como Ecuador y Perú (Barón, Clavijo y Combariza, 2010). 


\section{Resultados}

A raíz de la investigación se entiende que existe un afán por salvaguardar la tradición que abarcan estos alimentos. Por esta razón se realizó un recetario virtual con las distintas preparaciones que, sin dejar de lado la ancestralidad del producto; innova sobre la presentación del mismo. Por otro lado, se crea una página web que tiene como fin, no solo mostrar las distintas prelaciones; además, cuenta con un diverso contenido que busca promover el arraigo y amor por los productos autóctonos de la región andina.

\section{Página web.}

"Movimiento Andino" es una página web diseñada por estudiantes del programa de gastronomía de la Fundación Universitaria San Mateo. Como resultado, busca indagar e investigar por parte del internauta sobre los diferentes tubérculos sembrados en el país. Asimismo, dar a conocer las diferentes formas y métodos de preparación de estos.

Cuenta con una presentación en la cual el lector podrá instruirse acerca de cómo en el último siglo la cocina tradicional y ancestral ha perdido protagonismo al igual que los principales tubérculos abarcados en este sitio web como la papa ratona, las ibias y los ollucos.

Además de ello, cuenta con la ejemplificación de 14 recetas las cuales su ingrediente principal es uno de los tubérculos mencionados anteriormente. Así, demuestra al lector que este tipo de tubérculos tiene diferentes formas en las que pueden ser preparadas desde una bebida como lo es un masato, una mermelada o incluso en una arepuela.

Figura 1. Entorno de página web

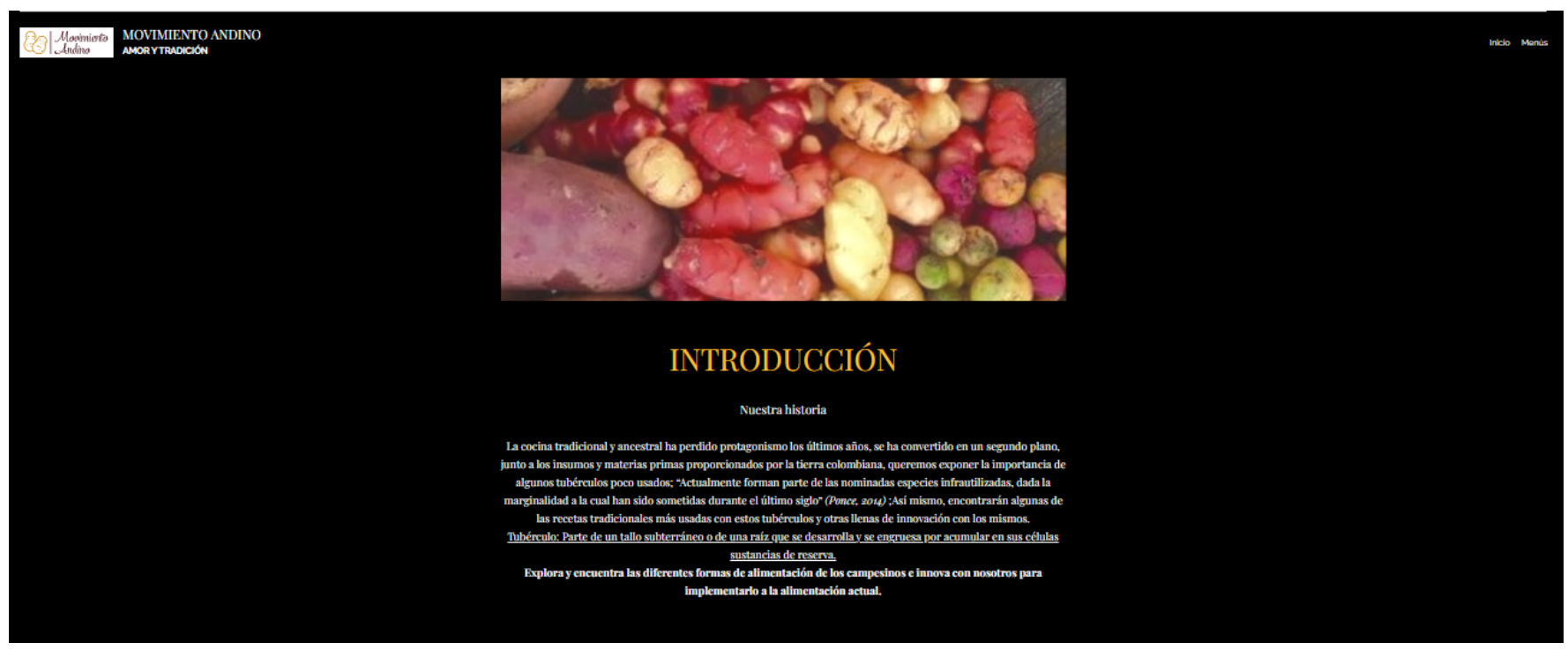

Fuente: Movimiento Andino, 2020. 
Figura 2. Entorno de página web

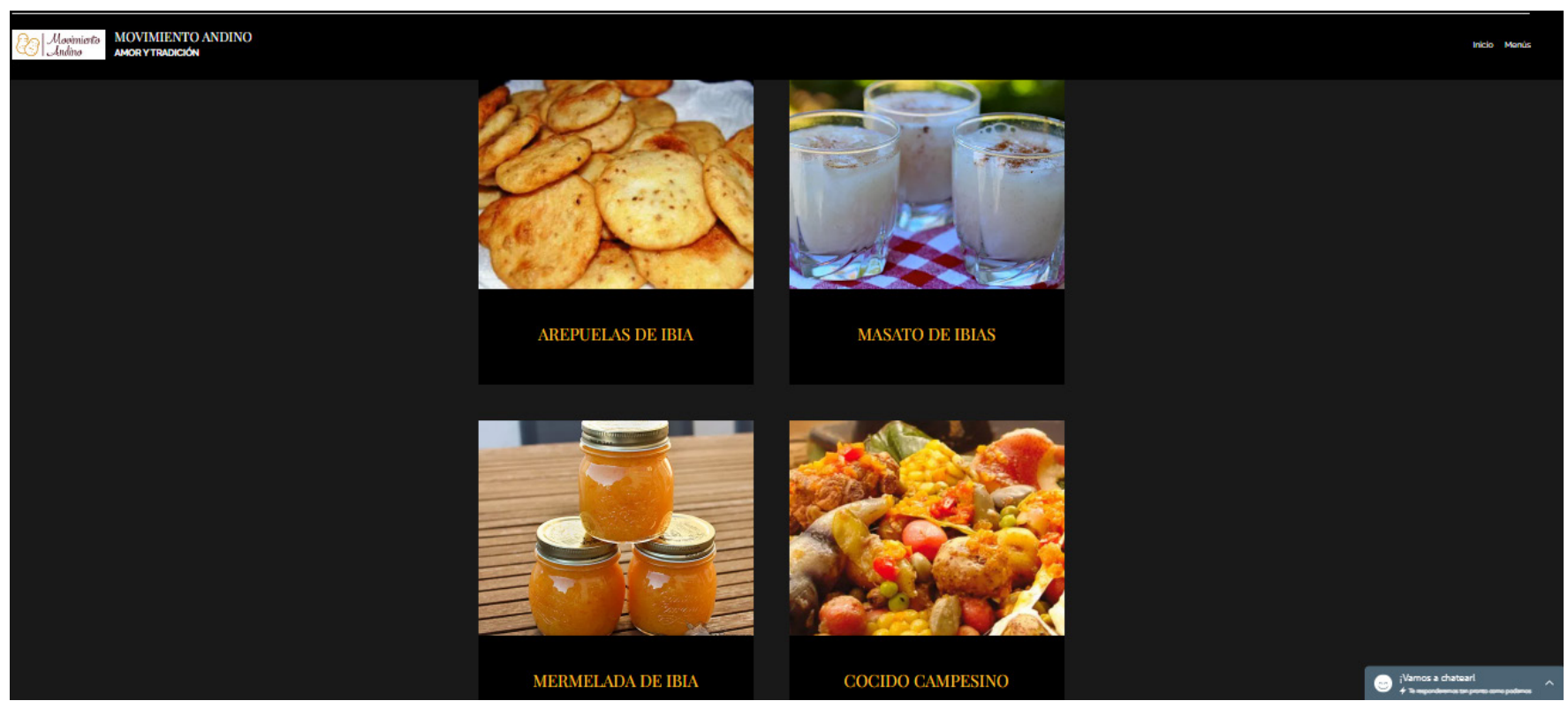

Fuente: Movimiento Andino, 2020.

\section{Recetario.}

El recetario andino surge a partir del desconocimiento de las diferentes formas de preparación de los tubérculos en el cual se encuentran variedad de preparaciones como bebidas, mermeladas entre otros. El recetario está constituido por un total de 18 recetas divididas en tres secciones de acuerdo a su ingrediente principal bien sean ibias, ollucos o papa ratona.

Figura 3. Entorno de página web
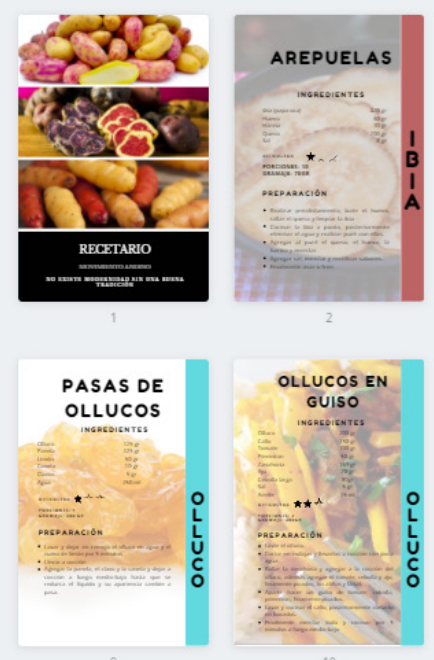
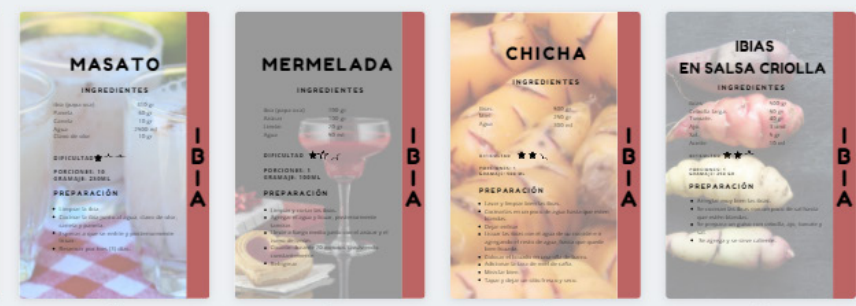
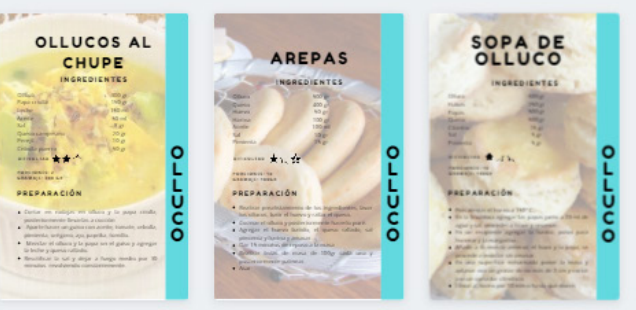
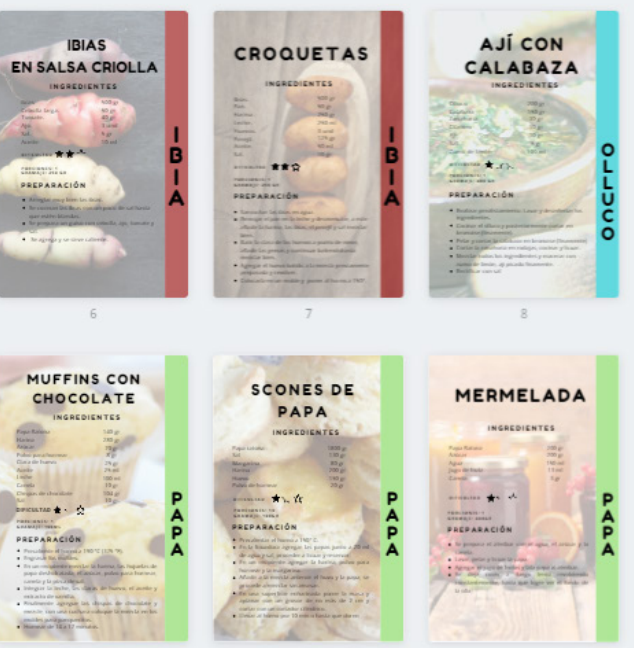

Fuente: Recetario Andino, 2020. 


\section{Conclusiones}

Dentro del desarrollo de la investigación fue fundamental el conocer la cadena de producción de las ibias y papa ratona, desde el conocimiento de los agricultores quienes a partir de sus experiencias identifican el valor cultural, que comprenden estos productos a la hora de elaborar recetas tradicionales de la región.

Por ello es fundamental incentivar la responsabilidad de gastrónomo profesional identificando los elementos de identidad que tienen los territorios en la generación de los productos tradicionales, estableciendo medios de difusión que ayuden a conocer las características de los tubérculos en el área andina.

Es necesario buscar alternativas que permitan diversificar el uso de las ibias, olluco, papa ratona u otros tubérculos, evitando la pérdida de cultivos importantes; no solo culturalmente, sino que además ayuden a mantener los suelos fértiles que caracterizan a la región.

Las recetas ancestrales, sobre los productos que se dan en la región de los Andes puede ser fuente de conocimiento, por ello se invita a la conservación de los valores ancestrales que existen en el territorio permitiendo crear canales de difusión de las diversas variedades de productos, mediante herramientas tecnológicas que ayuden a la salvaguardia de las tradiciones nacionales.

\section{Referencias bibliográficas}

Barón, M., Clavijo, N. y Combariza, J. (2010). Recetario Tubérculos andinos de turmequé y Ventaquemada de Boyacá, Colombia. Bogotá: Pontificia Universidad Javeriana. Recuperado de https://docplayer.es/3354047-Tuberculos-andinos-de-turmeque-y-ventaquemada-departamento-de-boyaca-colombia.html

Clavijo Ponce, N. L., y Pérez Martínez, M. E. (2014). Tubérculos andinos y conocimiento agrícola local en comunidades rurales de Ecuador y Colombia. Cuadernos De Desarrol/o Rural, 11(74), 149-166. https://doi.org/10.11144/ Javeriana.CRD11-74.taca

Daza, F. (2020). Movimiento Andino. Lfernandadaza. Recuperado de https:// Ifernandadaza.wixsite.com/movimientoandino

El Espectador. (20 de julio de 2017). El renacer de las papas nativas. El Espectador. Recuperado de https://www.elespectador.com/noticias/nacional/ el-renacer-de-las-papas-nativas/ 
Enríquez, D. (20 de Febrero de 2020). Habas y ollucos, alimentos de nuestras ancestros. (L. Rodríguez, Entrevistador).

Lescano, R. J. (1994). Genetica y mejoramiento de cultivos Altoandinos: Quinua, kañihua, tarwi, kiwicha, papa amarga, olluco, ashua y ocã. Puno, Perú: PIWA.

Movimiento Andino (2021). Recetario Movimiento Andino. Calameo. Recuperado de https://es.calameo.com/read/00533638205b09d1ced14

Rodríguez Arias, I. (2020). Conexión de las gastronomía con los eventos culturales en Bogotá. Sosquua 2(1), pp. 37-47

Rosero, M. (2010). Colección, caracterización y conservación de variabilidad genética de oca (Oxalis tuberosa Mol) en agroecosistemas paramunos del departamento de Nariño-Colombia (tesis de maestría). Universidad Nacional de Colombia, Palmira, Colombia. 